\title{
Management of Refractory Shallow Anterior Chamber Post Trabeculectomy
}

Meena Menon, Harshavardhan $\mathrm{VK}^{*}$

Department of Glaucoma Services, Sankara Eye Hospital, Bengaluru, India

DOI: $10.36347 /$ sjams.2020.v08i05.026

| Received: 09.05.2020 | Accepted: 16.05.2020 | Published: 19.05.2020

*Corresponding author: Dr. Harshavardhan VK

Abstract

Shallow anterior chamber (AC) with or without hypotony comprises one of the important post-operative complications of trabeculectomy surgery. The shallow anterior chamber may lead to secondary complications like synechiae formation, cataract progression, corneal endothelial decompensation, hypotony maculopathy, and persistent choroidal detachment. In this report, we describe a case of primary angle closure glaucoma that underwent trabeculectomy with MMC in which the post-operative period was complicated with refractory shallowing of AC presenting 4 weeks after trabeculectomy. This shallowing of AC didn't respond to conservative treatment and AC reformation with BSS. The patient was ultimately successfully treated with intracameral injection of Sodium Hyaluronate and Sulphur hexafluoride (SF6). This procedure can be a simple, safe, and effective treatment modality in such cases where conservative management has failed.

Keywords: Intracameral sulphur hexafluoride (SF6), Angle closure glaucoma, Hypotony, Trabeculectomy.

Copyright @ 2020: This is an open-access article distributed under the terms of the Creative Commons Attribution license which permits unrestricted use, distribution, and reproduction in any medium for non-commercial use (NonCommercial, or CC-BY-NC) provided the original author and source are credited.

\section{INTRODUCTION}

Shallow anterior chamber with or without hypotony comprises one of the important post-operative complications of trabeculectomy surgery $[1,2]$. It can be due to serous choroidal detachment, leaking bleb or overfiltration. Edmunds et al. [2] in a study of 1240 cases, found that $23.9 \%$ had shallow AC without iridocorneal touch in the first 2 postoperative weeks, $2.3 \%$ were found to have iridocorneal touch and $0.2 \%$ had corneolenticular touch. Shallow or flat anterior chamber may lead to secondary complications like development of synechiae, cataract progression, and corneal endothelial decompensation [3]. Prolonged hypotony may also lead to other vision affecting complications such as hypotony maculopathy and persistent choroidal detachment $[4,5]$. In this report, we describe a case of primary angle closure glaucoma who underwent trabeculectomy with MMC and further procedures to manage consequential refractory shallowing of anterior chamber.

\section{Case Description}

A 59-year-old lady complaining of unilateral, painful red eye for two days was referred for glaucoma management. She was on oral Acetazolamide therapy. On examination, vision was $6 / 6$ in her right eye and $6 / 36$ in her left eye. Anterior segment evaluation in her right eye revealed shallow iridocorneal angle in all four quadrants. There was corneal edema, closed angle with mid dilated, fixed pupil in her left eye. The intraocular pressures (IOP) were $18 \mathrm{~mm} \mathrm{Hg}$ and $66 \mathrm{~mm} \mathrm{Hg}$ in right eye and left eye respectively.

The patient was diagnosed as primary angle closure suspect in right eye and acute angle closure glaucoma in left eye. After control of IOP with oral Acetazolamide, topical Pilocarpine 2\% and intravenous Mannitol 20\%, the patient underwent $\mathrm{Nd}$ : YAG peripheral iridotomy in both eyes. She was then prescribed with topical Brimonidine $0.1 \%$ in left eye and Loteprednol $0.5 \%$ eyedrops in both eyes. The patient came for follow up after two days with patent iridotomy in both eyes, controlled IOP in right eye but high IOP in left eye (32 mm Hg). On Fundoscopy, right eye showed cup disc ratio of $0.4: 1$, with healthy and concentric neuroretinal rim, rest of the fundus details being normal. Left eye showed 0.6:1 cup disc ratio with neuroretinal rim thinning and bayonetting inferiorly, other fundus details were within normal limits. She was prescribed additional anti-glaucoma medications for her left eye including prostaglandin analogue and a beta blocker therapy. Patient was again examined after 1 week when her IOP was well controlled and patient was advised to continue the anti-glaucoma medications.

The patient reported again after 1 week with pain in her left eye with high IOP $(48 \mathrm{~mm} \mathrm{Hg})$ in spite of patent iridotomy and anti-glaucoma medications. She underwent Trabeculectomy with intra operative 
subconjunctival application of $0.2 \mathrm{mg} / \mathrm{mL}$ Mitomycin-C in her left eye for better IOP control. She was examined a week after the surgery which revealed mildly vascular diffuse filtering bleb in left eye with good IOP control $(10 \mathrm{~mm} \mathrm{Hg})$ without additional anti-glaucoma medications.

Patient presented again 4 weeks after trabeculectomy, complaining of pain in her left eye. On examination of left eye, cornea was clear, with grade II shallowing of anterior chamber with irido-corneal touch but no corneo-lenticular touch (Figure 1 and 2). Her IOP was $08 \mathrm{~mm} \mathrm{Hg}$, with large diffuse bleb. On Fundoscopy, there was $360^{\circ}$ choroidal effusion. She was treated with oral steroids and topical cycloplegic therapy. Five days later, the filtering bleb was large and diffuse with no leak, with IOP of $08 \mathrm{~mm} \mathrm{Hg}$. There was improvement on Fundoscopy, now with few shallow mounds of detectable choroidal detachments. Anterior chamber was still shallow for which she underwent anterior chamber reformation with balanced salt solution (BSS), with bandage contact lens (BCL) placement.

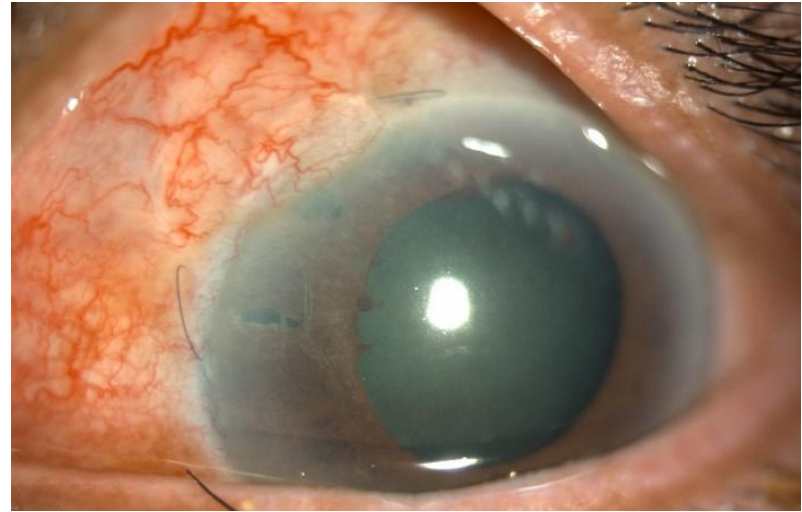

Fig-1: Left eye on diffuse illumination with clear cornea, large diffuse and vascular bleb. Both Nd:YAG peripheral iridotomy and surgical iridectomy near trabeculectomy ostium can be seen.

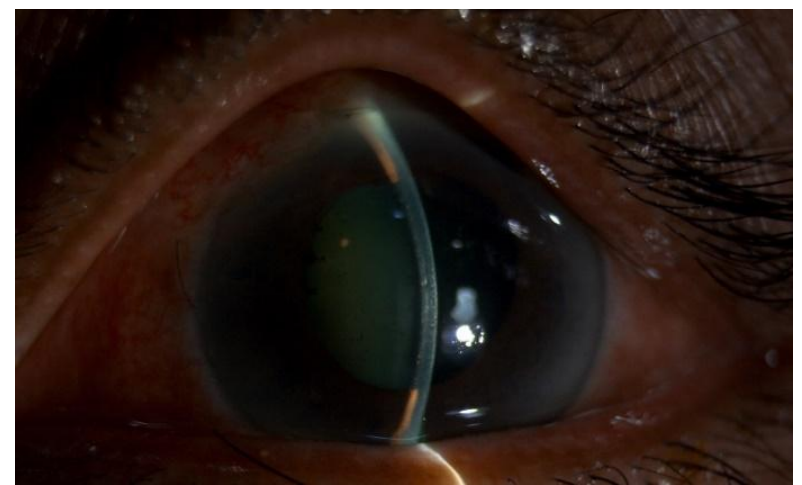

Fig-2: Left eye on slit illumination with shallow AC with iridocorneal touch but no corneo-lenticular touch as there is gap

between corneal endothelium and anterior lens surface.

A week later, examination in her left eye revealed few Descemet's membrane folds, persistent grade II shallowing of anterior chamber, BCL was in situ, and IOP was $10 \mathrm{~mm} \mathrm{Hg}$. Patient then underwent anterior chamber reformation with intracameral injection of $0.1 \mathrm{ml}$ each of Sodium Hyaluronate (1.4\%) and $20 \%$ Sulphur hexafluoride (SF6). In the immediate post procedure period, AC was formed well, with normal IOP. SF6 bubble was absorbed completely by $11^{\text {th }}$ day.

At last follow up, approximately 5 months after trabeculectomy surgery, AC was well formed with diffuse bleb with good IOP control (14 mm Hg). During course of management as well as follow up, cornea was clear, although there was a noticeable progression in the cataractous lens changes, but not warranting immediate cataract extraction. Fundoscopy of right eye showed cup disc ratio of $0.4: 1$, with healthy and concentric neuroretinal rim, rest of the fundus details being normal. Left eye showed 0.7:1 cup disc ratio with mild progression in inferior neuroretinal rim thinning and other fundus details were within normal limits.

\section{DISCUSSION}

Shallowing of the AC is common complication following trabeculectomy and most of these cases can be managed conservatively $[6,7,8]$. If optimal $\mathrm{AC}$ depth is not restored, IOP control will be lost secondary to obliteration of the $\mathrm{AC}$ angle by peripheral anterior synechiae, or by failure of development of a bleb [7]. In addition, aqueous misdirection may occur as a result of cilio-lenticular block, resulting in malignant glaucoma.

Austin et al. [9] reported the increased incidence of post-operative shallow $\mathrm{AC}$ in cases of angle closure glaucoma as well as those with preoperative shallow anterior chamber. Li et al. [10] reported angle closure glaucoma, neovascular glaucoma, refractory high IOP following primary glaucoma surgery, and higher IOP at admission carry higher risk for incidence of grade III flat anterior chamber after glaucoma filtration surgery.

Several successful treatment modalities have been suggested for managing shallow AC after trabeculectomy. Austin et al. in their study managed post-operative shallow AC using medical treatment, intracameral injection of BSS, visco-elastic devices, gases like air, sulphur hexafluoride (SF6) [9]. Weinstein et al. used SF6. [11] de Barros et al. in their study compared the efficiency of visco-elastics, injection of BSS with drainage of choroidal effusion and conservative management [12]. Wilson et al. successfully performed choroidal tap along with intracameral injection of perfluoropropane (C3F8) [13]. Franks et al. in their study, suggested injection of C3F8 as an alternative modality of treating post-operative shallow AC [14]. Gotzaridis et al. in their case series reported promising results in managing post-operative hypotony by using SF6 [15].

We prefer SF6 since it lasts in the AC for an optimal period of time, it remains as a single bubble, deepening the AC until aqueous gradually replaces it. 
Although corneal toxicity with SF6 has been previously reported [16] our patient did not develop clinically detectable corneal complications. However, there was a mild progression in the cataract at 5 months follow up, not warranting immediate cataract extraction.

\section{Conclusion}

Filtration surgery done especially for primary angle closure glaucoma can be associated with refractory shallowing of anterior chamber depth postoperatively. Intracameral injection of visco-elastic devices like Sodium Hyaluronate and Sulphur hexafluoride can be simple, safe and effective treatment modality in such cases where conservative management has failed.

\section{REFERENCES}

1. Jampel HD, Musch DC, Gillespie BW, Lichter PR, Wright MM, Guire KE, Collaborative Initial Glaucoma Treatment Study Group. Perioperative complications of trabeculectomy in the collaborative initial glaucoma treatment study (CIGTS). American journal of ophthalmology. 2005 Jul 1;140(1):16-22.

2. Edmunds B, Thompson JR, Salmon JF, Wormald RP. The National Survey of Trabeculectomy. III. Early and late complications. Eye (Lond). 2002;16(3):297-303. doi: 10.1038/sj.eye.6700148

3. Stewart WC, Shields MB. Management of anterior chamber depth after trabeculectomy. Am J Ophthalmol. 1988;106(1):41-44.

4. Gass KK. Hypotony maculopathy. In: Bellows JG, ed. Contemporary Ophthalmology. Baltimore: Williams-Wilkins; 1972:34.

5. Cohen SM, Flynn HW Jr, Palmberg PF, Gass JD, Grajewski AL, Parrish RK 2nd. Treatment of hypotony maculopathy after trabeculectomy. Ophthalmic Surg Lasers. 1995;26(5):435-441.

6. Mills KB. Trabeculectomy: a retrospective longterm follow-up of 444 cases. Br J Ophthalmol. 1981;65(11):790-795.
7. Aminlari A. Postoperative complications of glaucoma filtering surgery. Glaucoma. 1990; 12: 101-1 10.

8. Kao SF, Lichter PR, Musch DC. Anterior chamber depth following filtration surgery. Ophthalmic Surg. 1989;20(5):332-336.

9. Austin MW, Wishart PK. Reformation of the anterior chamber following trabeculectomy. Ophthalmic Surg. 1993;24(7):461-466.

10. Li XJ, Filek R, He XG, Wang W, Liu H, He L, Tang YR, Xie L. Risk factors for flat anterior chamber after glaucoma filtration surgery. International journal of ophthalmology. 2018;11(8):1322.

11. Weinstein O, Oshry T, Tessler Z, Lifshitz T. Use of sulphur hexafluoride for anterior chamber reformation following trabeculectomy. British journal of ophthalmology. 1998 Oct 1;82(10):1220-

12. de Barros DS, Navarro JB, Mantravadi AV, Siam GA, Gheith ME, Tittler EH, Baez KA, Martinez SM, Spaeth GL. The early flat anterior chamber after trabeculectomy: a randomized, prospective study of 3 methods of management. Journal of glaucoma. 2009 Jan 1;18(1):13-20.

13. Wilson MR, Yoshizumi MO, Lee DA, Martin W, Higginbotham EJ. Use of intraocular gas in flat anterior chamber after filtration surgery. Archives of Ophthalmology. 1988 Oct 1;106(10):1345-.

14. Franks WA, Hitchings RA. Intraocular gas injection in the treatment of cornea-lens touch and choroidal effusion following fistulizing surgery. Ophthalmic Surg. 1990;21(12):831-834.

15. Gotzaridis SV, Portaliou DM, Mela VA, Xanthopoulou P, Kymionis GD. Anterior chamber sulfur hexafluoride (SF6) injection for the management of postsurgery hypotony in glaucoma patients. Journal of glaucoma. 2018 May $1 ; 27(5): 476-9$.

16. Lee DA, Wilson MR, Yoshizumi MO, Hall M. The ocular effects of gases when injected into the anterior chamber of rabbit eyes. Arch Ophthalmol. 1991;109(4):571-575. 